\title{
A report of two cases of bulbospinal form Alexander disease and preliminary exploration of the disease
}

\author{
XIAOXUAN SONG ${ }^{1 *}$, JINGWEN JIANG $^{1 *}$, WOTU TIAN ${ }^{1}$, FEIXIA ZHAN ${ }^{1}$, \\ ZEYU ZHU ${ }^{1}$, BINYIN LI $^{1}$, HUIDONG TANG ${ }^{1}$ and LI CAO $^{1,2}$ \\ ${ }^{1}$ Department of Neurology, Rui Jin Hospital, Shanghai Jiao Tong University School of Medicine, Shanghai 200025;
${ }^{2}$ Department of Neurology, Shanghai Jiao Tong University Affiliated Sixth People's Hospital, Shanghai 200233, P.R. China
}

Received December 15, 2020; Accepted May 10, 2021

DOI: $10.3892 / \mathrm{mmr} .2021 .12211$

\begin{abstract}
Alexander disease (AxD) is a cerebral white matter disease affecting a wide range of ages, from infants to adults. In the present study, two cases of bulbospinal form AxD were reported, and a preliminary exploration of $\mathrm{AxD}$ was conducted thorough clinical, functional magnetic resonance imaging (fMRI) and functional analyses. In total, two de novo mutations in the glial fibrillary acidic protein $(G F A P)$ gene (c.214G $>$ A and c.1235C $>$ T) were identified in unrelated patients (one in each patient). Both patients showed increased regional neural activity and functional connectivity in the cerebellum and posterior parietal cortex according to fMRI analysis. Notably, grey matter atrophy was discovered in the patient with c. $214 \mathrm{G}>\mathrm{A}$ variant. Functional experiments revealed aberrant accumulation of mutant GFAP and decreased solubility of c. $1235 \mathrm{C}>\mathrm{T}$ variant. Under pathological conditions, autophagic flux was activated for GFAP aggregate degradation. Moreover, transcriptional data of AxD and healthy human brain samples were obtained from the Gene Expression Omnibus database. Gene set enrichment analysis revealed an upregulation of immune-related responses and downregulation of ion transport, synaptic transmission and neurotransmitter homeostasis. Enrichment analysis of cell-specific differentially expressed genes also indicated a marked inflammatory environment in AxD. Overall, the clinical features of the two patients with bulbospinal form AxD were thoroughly described. To the best of our knowledge, the brain atrophy pattern and spontaneous brain functional network activity of patients with AxD were
\end{abstract}

Correspondence to: Dr Li Cao or Dr Huidong Tang, Department of Neurology, Rui Jin Hospital, Shanghai Jiao Tong University School of Medicine, 197 Ruijin 2nd Road, Huangpu, Shanghai 200025, P.R. China

E-mail: caoli2000@yeah.net

E-mail: thd10495@rjh.com.cn

${ }^{*}$ Contributed equally

Key words: Alexander disease, gene mutations, brain network, functional study, inflammation explored for the first time. Cytological experiments provided evidence of the pathogenicity of the identified variants. Furthermore, bioinformatics analysis found that inflammatory immune-related reactions may play a critical role in $\mathrm{AxD}$, which may be conducive to the understanding of this disease.

\section{Introduction}

Alexander disease $(\mathrm{AxD})$ is a progressive and fatal neurological disorder characterized by astrocytic cytoplasmic inclusions (1). These inclusions, namely Rosenthal fibers, contain glial fibrillary acidic protein (GFAP) along with several stress proteins, such as small heat shock proteins 27 and $\alpha \mathrm{B}$-crystallin (2). In 2001, GFAP was identified as a candidate gene for $\mathrm{AxD}$, which encodes the major intermediate filament (IF) protein in astrocytes (3). GFAP plays an important role in cell migration, motility and mitosis, and has also been implicated in the mechanical integrity of cells and cell signaling (4). Unlike most variants of other IF disorders, which act in a loss-of-function manner, all known GFAP mutations in AxD are genetically dominant and appear to produce a toxic gain-of-function effect (5). The downstream consequences include the sequestration of protein chaperones, the abnormality of IF network assembly and the predisposition for the GFAP protein to form aggregates, as well as the hyperactivation of cellular stress (6). The combination of these consequences then induces numerous dysfunctions, from intracellular vesicle regulation to ion homeostasis, to synapses formation and cellular communication, ultimately causing neurological disorders (7). However, the specific mechanism of $\mathrm{AxD}$ pathogenesis remains unclear.

In terms of clinical characteristics, $\mathrm{AxD}$ is typically classified into three forms, according to age at onset: The infantile ( $<2$ years old), juvenile (2-12 years old) and adult ( $\geq 13$ years old) forms (8). However, the heterogeneity of neurological manifestations and wide variety in onset age render diagnosis challenging. Based on neurological analysis and magnetic resonance imaging (MRI), Yoshida et al (9) proposed new guidelines for diagnosing AxD in 2011. Under these guidelines, AxD can be classified into three types: The cerebral (type 1), bulbospinal (type 2) and intermediate (type 3 ) forms. The cerebral form $\mathrm{AxD}$ is characterized by delayed psychomotor development, convulsions, macrocephaly and 
leukoencephalopathy, appearing as frontal lobe predominance on brain imaging scans. Patients with the bulbospinal (type 2) form present with muscle weakness, hyperreflexia and distinct bulbar dysfunction, typically appearing as medulla oblongata or cervical cord atrophy on MRI scans. The intermediate (type 3 ) form is characterized by several of the symptoms of the cerebral and bulbospinal forms. Although featured neurological and neuroradiological findings can assist the diagnosis of $\mathrm{AxD}$, the definitive diagnosis currently relies on a genetic test or pathological examination (10).

The present study reported two cases of bulbospinal form AxD, and clinical, functional (f)MRI and functional analyses were conducted. In addition, bioinformatics analysis of published data was performed to explore the potential pathogenic mechanisms of AxD.

\section{Materials and methods}

Participants. In total, two identified probands (P3433 and P4288) with AxD from two unrelated families and 500 healthy subjects as controls for genetic analysis, as well as 15 normal individuals as controls for imaging analysis were enrolled from Department of Neurology, Rui Jin Hospital, Shanghai Jiao Tong University School of Medicine (Shanghai, China). Diagnostic workups of patients with AxD included taking history, physical examination and brain imaging according to diagnosis guidelines (9). Patient P3433 was a 29-year-old female (recruited March 2018). Patient P4288 was a 33-year-old man (recruited December 2018). The healthy subjects who did not carry any disease and had no family history of AxD were enrolled from March 2018 to May 2019. For genetic analysis, the 500 healthy controls (234 females and 266 males; age, 15-47 years; mean age, 34.570 $\$ 9.079$ years). For imaging analysis, 15 normal controls were recruited from January 2019 to May 2019 (10 females and 5 males; age, 23-45 years; mean age, $30.4 \pm 7.5)$. This study was approved by the Ethics Committee of Rui Jin Hospital, Shanghai Jiao Tong University School of Medicine (approval no. 2019-153; Shanghai, China). All participants provided written informed consent for participation.

Genetic analysis. Genomic DNA was extracted from peripheral blood using the phenol-chloroform method (11). EDTA anticoagulated blood and red blood cell lysis buffer $(10 \mathrm{mmol} / \mathrm{l}$ $\mathrm{NaCl}, 10 \mathrm{mmol} / \mathrm{l}$ Tris- $\mathrm{HCl}, 5 \mathrm{mmol} / \mathrm{MgCl}_{2}$ ) were mixed and incubated at $4^{\circ} \mathrm{C}$ for $20 \mathrm{~min}$, then centrifuged at $1,811 \mathrm{x} \mathrm{g}$ for $10 \mathrm{~min}$ at $4^{\circ} \mathrm{C}$. The cell lysates were digested with nuclei lysis buffer ( $5 \mathrm{mmol} / 1 \mathrm{NaCl}, 10 \mathrm{mmol} / 1$ EDTA, $10 \mathrm{mmol} / 1$ Tris-HCl), $10 \%$ SDS and protease K solution $(20 \mathrm{mg} / \mathrm{ml}$; Thermo Fisher Scientific, Inc.) at $37^{\circ} \mathrm{C}$ overnight. After digestion, equal volumes of tris-saturated phenol and chloroform/isopropanol mixture (24:1) were added to each tube. Following centrifugation at $1,811 \mathrm{x} \mathrm{g}$ for $10 \mathrm{~min}$ at $4^{\circ} \mathrm{C}$, the upper aqueous phase was transferred to new tubes and mixed gently with 2 volumes of room temperature absolute ethanol to precipitate the DNA. After rinsing the DNA pellet with $75 \%$ ethanol twice (3,220 x g, $1 \mathrm{~min})$, the supernatant was discarded and the DNA pellet was air dried. Then, the DNA was dissolved in TE buffer for $2 \mathrm{~h}$ at $37^{\circ} \mathrm{C}$ and stored at $-80^{\circ} \mathrm{C}$. Whole-exome sequencing was performed in two probands. DNA quality was verified by the 2200 TapeStation system (Agilent Technologies, Inc.). A total of $3 \mu \mathrm{g}$ DNA per sample was utilized for WES using SureSelectXT Human All Exon V6 kits (cat. no. 5190-8864; Agilent Technologies, Inc.) according to the manufacturer's protocol. The concentration and quality of DNA libraries were detected by Qubit 3.0 Fluorometer (Thermo Fisher Scientific, Inc.) and 2200 TapeStation system (Agilent Technologies, Inc.). Using a loading concentration of $2 \mathrm{nM}$, data were generated by 150 base paired-end reads on an HiSeq X Ten platform (Illumina, Inc.) using Hiseq X HD Reagent V2.5 kit (cat. no. FC-501-2501; Illumina, Inc.). The sequence reads were aligned to the human genome reference sequence (GRCh37/hg19) with BWA-MEM software version 0.7.17 (12). Variant calling and annotation were performed by Genome Analysis Toolkit (GATK version 4.1.9.0) software and Annotate Variation (ANNOVAR version 20191024) software, respectively $(13,14)$. Variants in which the minor allele frequency was $>1 \%$ were filtered using public databases, including 1000 Genomes (1000 g; internationalgenome.org), The Exome Aggregation Consortium (Ex AC; gnomad.broadinstitute.org) and The Genome Aggregation Database (gnomAD; gnomad. broadinstitute.org). PolyPhen-2 (http://genetics.bwh.harvard. edu/pph2), Scale-invariant feature transform (SIFT; http://sift. jcvi.org) and MutationTaster (http://www.mutationtaster.org) were used for pathogenic prediction. The variants were further interpreted and classified according to the American College of Medical Genetics and Genomics (ACMG) guidelines (15). Putative pathogenic variants were subsequently confirmed by Sanger sequencing, and a total of 500 healthy subjects were enrolled as controls. All GFAP variants were denoted as RefSeq NM_002055.5. In addition, all previously reported mutations of the GFAP gene were summarized and labeled in the diagram of GFAP protein domain structure according to the Human Gene Mutation Database (hgmd.cf.ac.uk/).

MRI acquisition, preprocessing and statistical analysis. The two probands were scanned on an MR system (Ingenia, Philips 3T MR system; Philips Healthcare) with an 8-channel head coil array, using 15 normal individuals as the controls. The protocol included a three-dimensional high-resolution turbo field echo T1-weighted sequence for neuroanatomy (sagittal slice orientation; matrix $=256 \times 256$; repetition time $=7.2 \mathrm{msec}$; echo time $=3.3 \mathrm{msec}$; flip angle $=7^{\circ}$; slice thickness $=1 \mathrm{~mm}$; slice number $=192$ ). Resting-state blood oxygen level-dependent MRI used T2*-weighted echo-planar imaging sequence (240 functional images; sagittal slice orientation; 39 slices; slice thickness $=3.5 \mathrm{~mm}$; matrix $=64 \times 64$; repetition time $=2,000 \mathrm{msec}$; echo time $=30 \mathrm{msec}$; flip angle $=90^{\circ}$ ). The two patients also underwent T2-weighted fluid-attenuated inversion recovery to obtain a more accurate image of white matter lesions.

The T1-weighted anatomical image was first segmented into grey matter, white matter and cerebrospinal fluid using computational anatomy toolbox (CAT)12 (http://www.neuro. uni-jena.de) in Statistical Parametric Mapping (SPM)12 software (v.6685; http://fil.ion.ucl.ac.uk/spm) in a MATLAB 2014b environment (https://www.mathworks.com), with reference to tissue probabilistic maps in Montreal Neurological Institute (MNI) space. White matter hyperintensity was also estimated using a grey matter-white matter tissue probability 
A

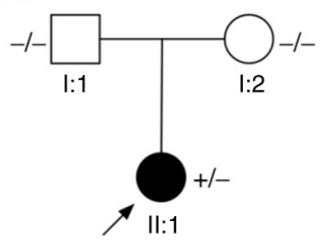

c.214G $>A(p . E 72 K)$

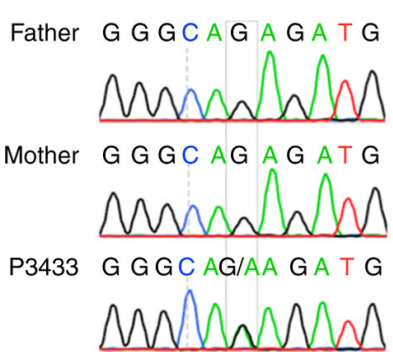

Father G G G C A G A G T G

B

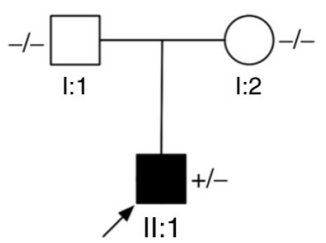

c. $1235 \mathrm{C}>\mathrm{T}($ p.T412l)

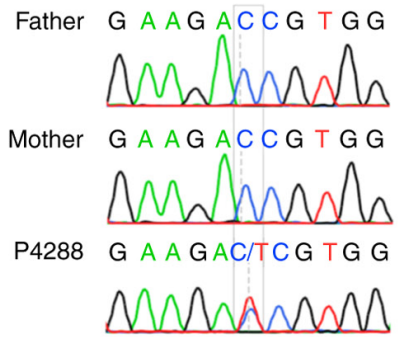

C

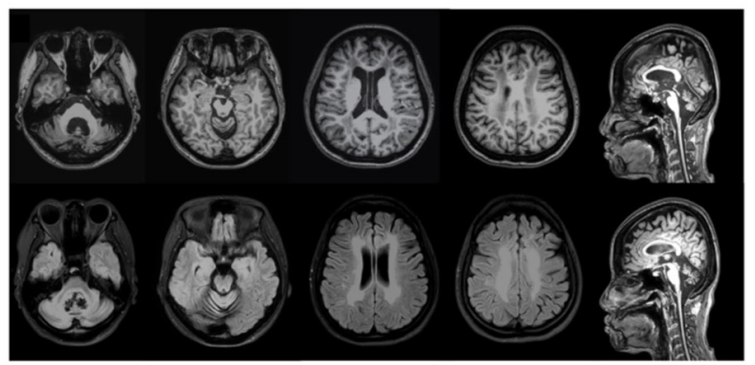

$\mathrm{D}$

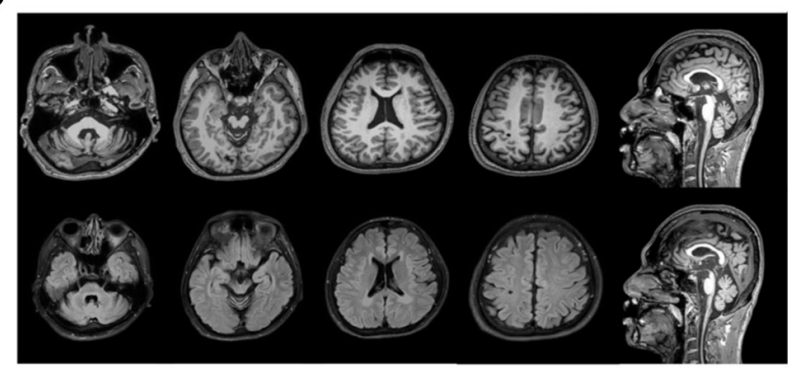

E

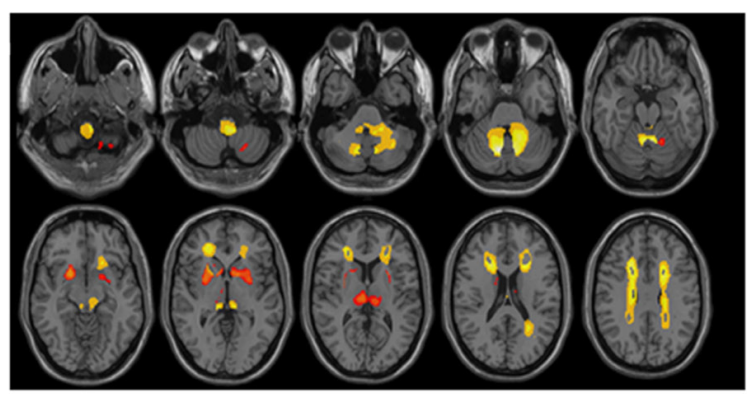

G

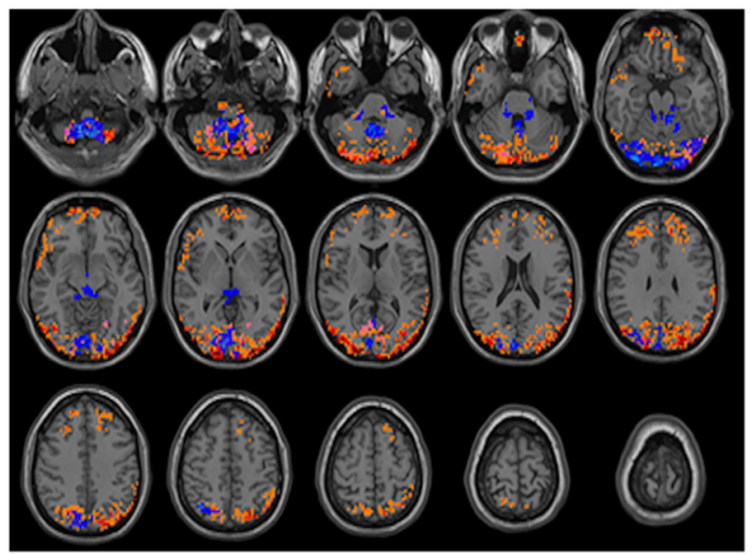

F

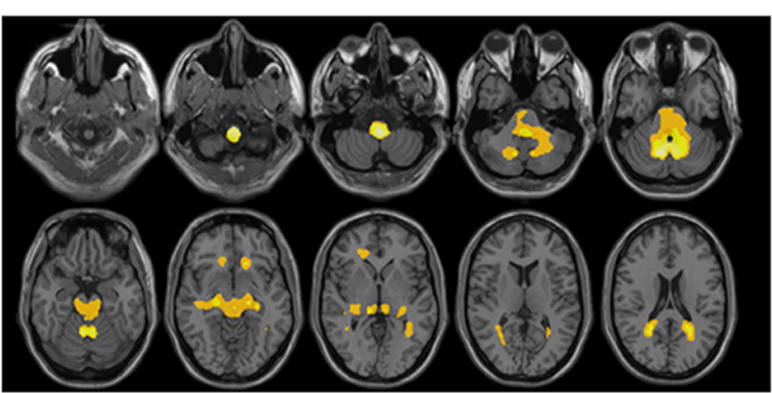

$\mathrm{H}$

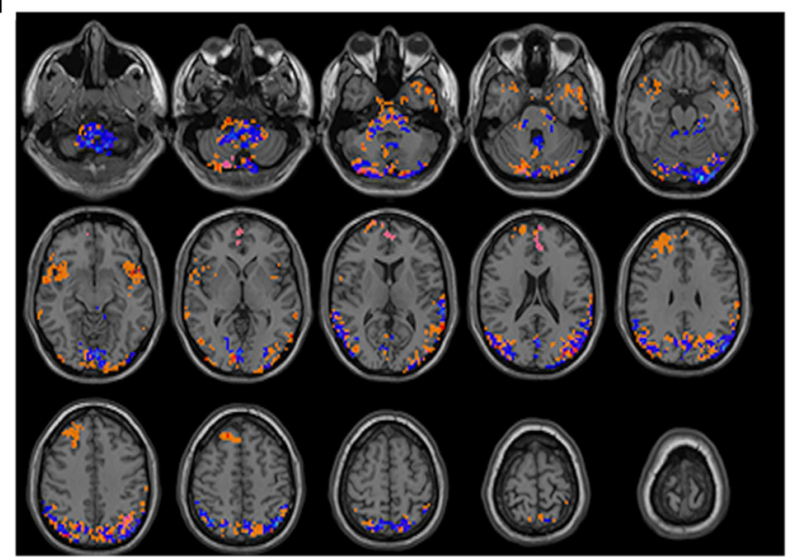

Figure 1. Genetic and imaging analysis of two probands. Pedigrees and corresponding chromatograms for patients (A) P3433 and (B) P4288. T2-weighted fluid attenuated inversion recovery images of patients (C) P3433 and (D) P4288. White and grey matter analysis of patients (E) P3433 and (F) P4288. Red/orange, significantly larger volume of grey matter in normal controls than in the patient; yellow, significantly larger volume of white matter in normal controls than in the patient. Analysis of neural activity and functional connectivity of patients (G) P3433 and (H) P4288. Red/orange, voxels with a significantly higher degree centrality; blue, voxels with significantly higher amplitude of low-frequency fluctuation; pink, voxels with a significantly higher amplitude of regional homogeneity.

map in CAT12. Voxel-based morphometry was performed between each patient and normal controls to analyze for grey and white matter, using unpaired Student's t-tests with total intracranial volume (TIV) as a covariate. False discovery rate (FDR) correction was used to correct for multiple comparisons at an FDR-adj.P $<0.05$.

With regards to the fMRI, the first 40 fMRI images of each individual were discarded. The remaining 200 images were realigned to adjust head motion, co-registered to the anatomical image and normalized to the MNI space using a modified MATLAB toolbox [Data Processing \& Analysis of Brain Imaging (DPABI); version 3.0] (16). Mean amplitude of low-frequency fluctuation (ALFF) was computed to represent regional neural activity of the individuals, regional homogeneity (ReHo) and degree centrality (DC) to represent the quantity of functional connections of a region (17-19). 


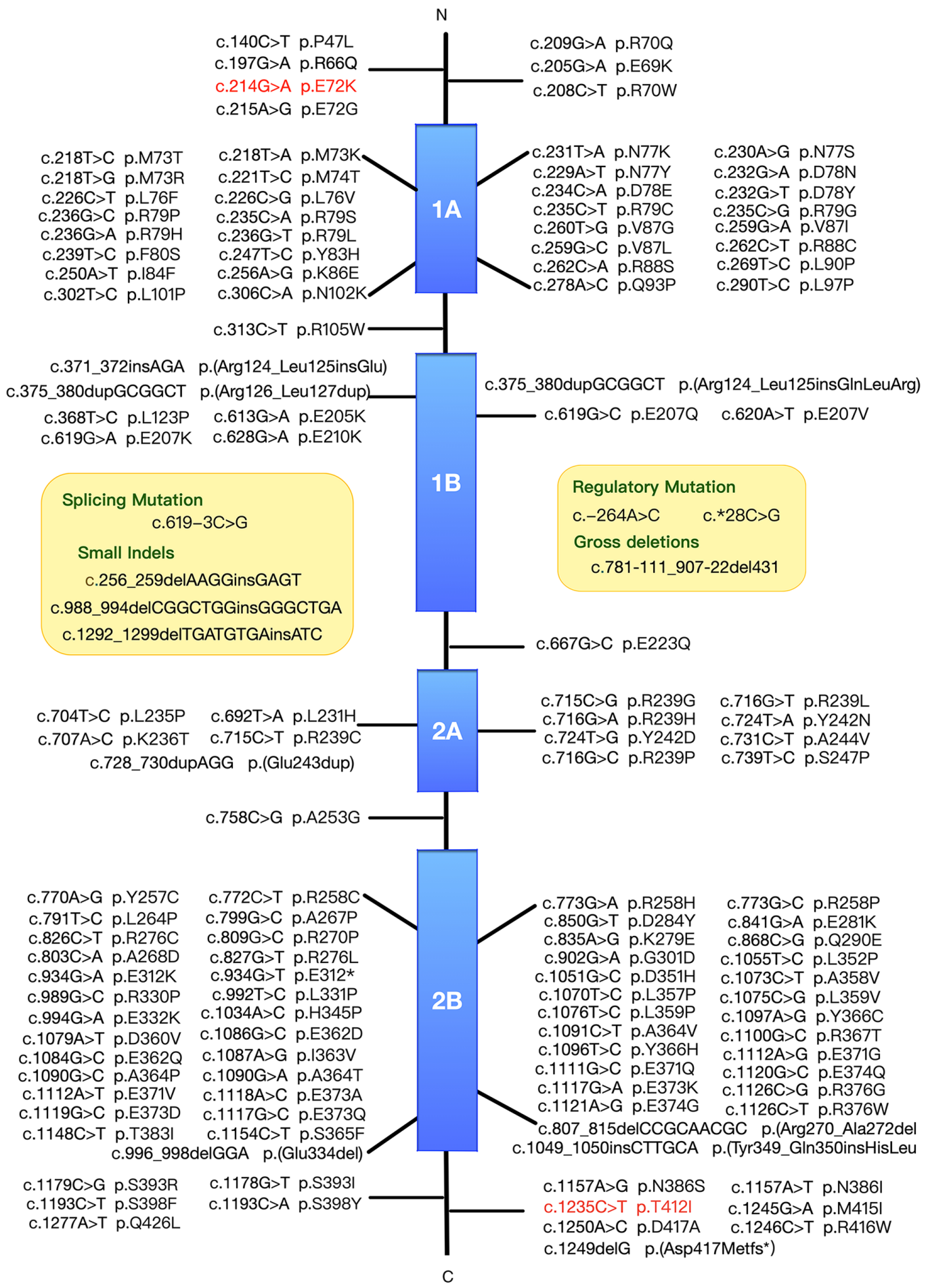

Figure 2. Schematic diagram of GFAP structure with identified variants. Mutations identified in the present study are in red font. GFAP, glial fibrillary acidic protein.

Individual participants' weighted DC values were obtained from all voxels in standard space using DPABI. Mean ALFF and voxel-wise centrality values were also compared between each patient and the controls using unpaired Student's t-tests with FDR-adj.P $<0.05$, with voxel-based morphometry of grey matter volume as a covariate.

Cell culture and transfection. All plasmids were purchased from GeneCreate. cDNA of wild-type (WT) or mutant (MUT) GFAP (NM_002055.5) was inserted into the
pcDNA3.1-green fluorescent protein (GFP) plasmids to express GFP-tagged fusion proteins. The 293T cell line was obtained from The Cell Bank of Type Culture Collection of The Chinese Academy of Sciences. 293T cells were grown in DMEM (Gibco; Thermo Fisher Scientific, Inc.) supplemented with 10\% FBS (Gibco; Thermo Fisher Scientific, Inc.) and $1 \%$ penicillin-streptomycin (Invitrogen; Thermo Fisher Scientific, Inc.) at $37^{\circ} \mathrm{C}$ in a humidified incubator with $5 \% \mathrm{CO}_{2}$. Next, $2 \times 10^{5}$ cells/well were seeded into 6 -well plates or $5 \times 10^{4}$ cells/well were seeded into 24 -well plates for 

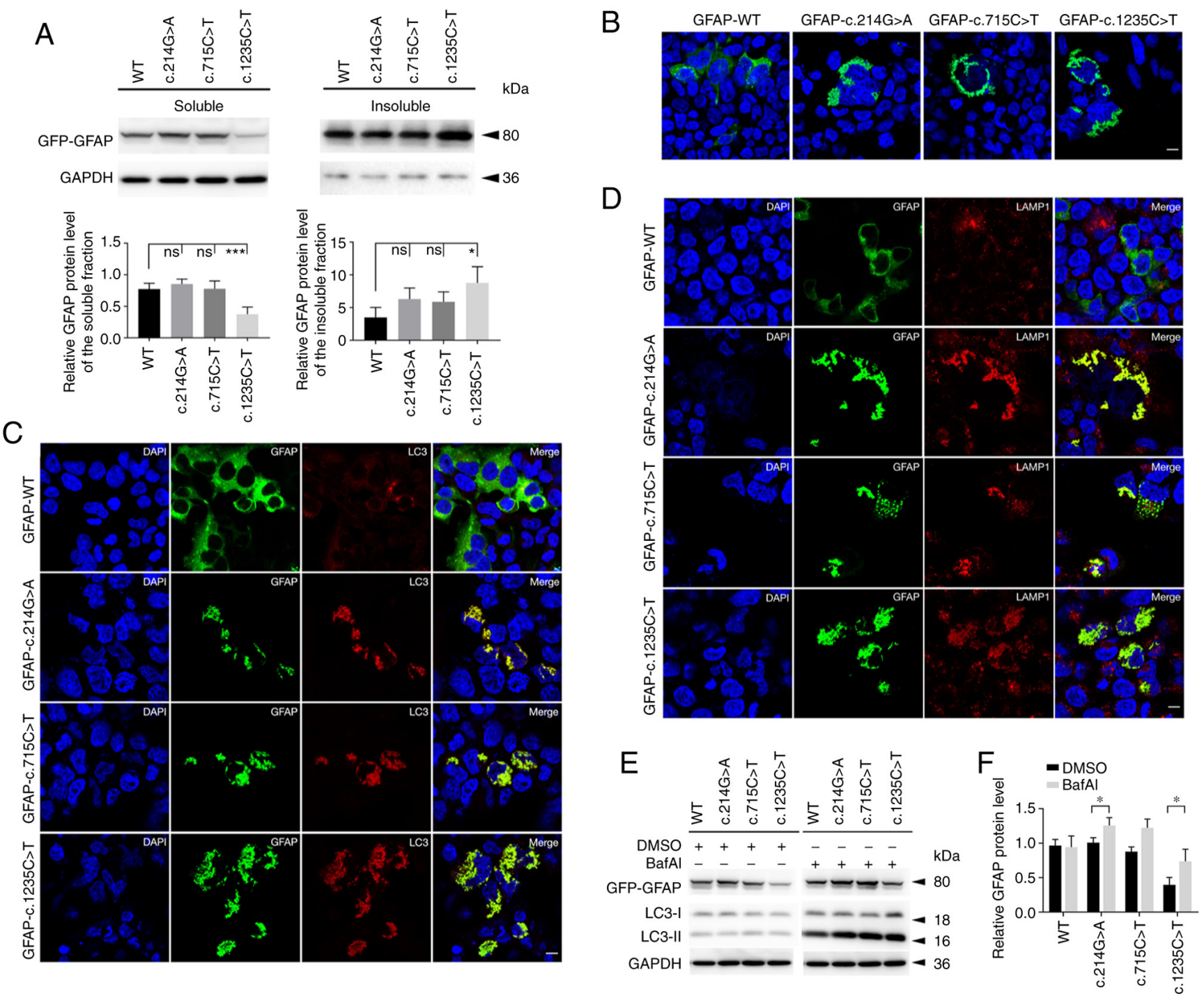

Figure 3. Functional analysis of mutant GFAP. (A) Relative protein levels of GFAP in the soluble and insoluble fractions [one-way ANOVA followed by Tukey's test; bar plot indicates the statistical analysis (mean \pm SD) of three independent experiments; ${ }^{*} \mathrm{P}<0.05$ and $\left.{ }^{* * *} \mathrm{P}<0.001\right]$. (B) Immunofluorescence images showing the distribution and morphology of GFAP proteins. Scale bar, $10 \mu \mathrm{m}$. (C and D) Co-localization analysis of GFAP proteins and (C) LC3 or (D) LAMP1 by immunostaining. Scale bar, $10 \mu \mathrm{m}$. (E) Detection of the soluble levels of GFAP and LC3-II under BafAI treatment by western blot analysis. (F) Statistical analysis of GFAP levels of (E) [paired Student's t-test; bar plot indicates the statistical analysis (mean \pm SD) of three independent experiments; ${ }^{*} \mathrm{P}<0.05$ ]. ns, non-significant; GFAP, glial fibrillary acidic protein; LAMP1, lysosomal-associated membrane protein 1; LC3, autophagy light chain 3; BafAI, bafilomycin AI; WT, wild-type; GFP, green fluorescent protein.

transfection. Then, $24 \mathrm{~h}$ after plating, 293T cells were transiently transfected with WT or MUT GFAP-GFP (c.214G>A and c. $1235 \mathrm{C}>\mathrm{T}$ ) plasmids using Lipofectamine ${ }^{\circledR} 3000$ transfection reagent (Invitrogen; Thermo Fisher Scientific, Inc.) at room temperature. A hot-spot mutation (c.715C >T, p.R239C) was set as the positive control (8). All experiments were independently repeated three times.

Western blotting and immunofluorescence. A total of $48 \mathrm{~h}$ after transfection, 293T cells in 6-well plates were collected to extract proteins for western blot analysis. For lysosomal inhibitor treatment, bafilomycin AI (BafAI; 5 nM; Merck KGaA) was added to $293 \mathrm{~T}$ cells $24 \mathrm{~h}$ post-transfection, with DMSO as the vehicle control. $293 \mathrm{~T}$ cells were then incubated for $12 \mathrm{~h}$ to extract proteins (20). Protein concentrations were quantified using Pierce BCA Protein Assay kit (cat. no. 23225; Thermo Fisher Scientific, Inc.). RIPA buffer (Beyotime Institute of Biotechnology) with protease inhibitors was used for protein extraction. Following centrifugation at $13,000 \mathrm{x}$ g for $20 \mathrm{~min}$ at $4^{\circ} \mathrm{C}$, cell lysates were separated into two parts, the supernatant as the soluble fraction and the sedimentation as the insoluble fraction. The insoluble fraction was dissolved with denaturing protein solubilization reagents (Invent Biotechnologies, Inc.). A total of $20 \mu \mathrm{g}$ protein was loaded per lane. Proteins were separated via $10 \%$ SDS-PAGE, and then subsequently transferred to a PVDF membrane. The membrane was blocked with 5\% BSA (Sangon Biotech Co., Ltd.) for $60 \mathrm{~min}$ at room temperature. Anti-GFP (1:2,500; cat. no. GFP-1010; Aves Labs, Inc.), anti-autophagy light chain 3 (LC3; 1:1,000; cat. no. 3868; Cell Signaling Technology, Inc.) and anti-lysosomal-associated membrane protein 1 (LAMP-1) antibodies (1:1,000; cat. no. 9091; Cell Signaling Technology, Inc.) were used to detect relative protein expression levels. GAPDH antibodies (1:1,000; cat. no. 2118; Cell Signaling Technology, Inc.) 
A

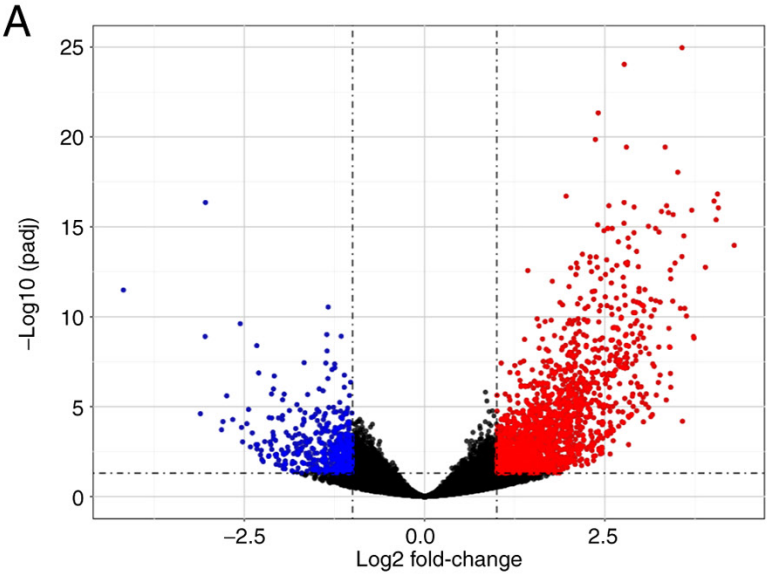

C

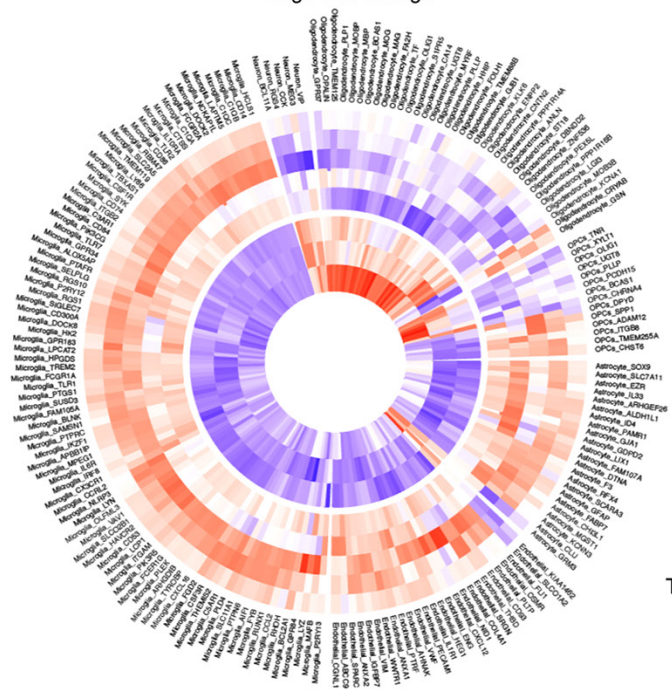

E

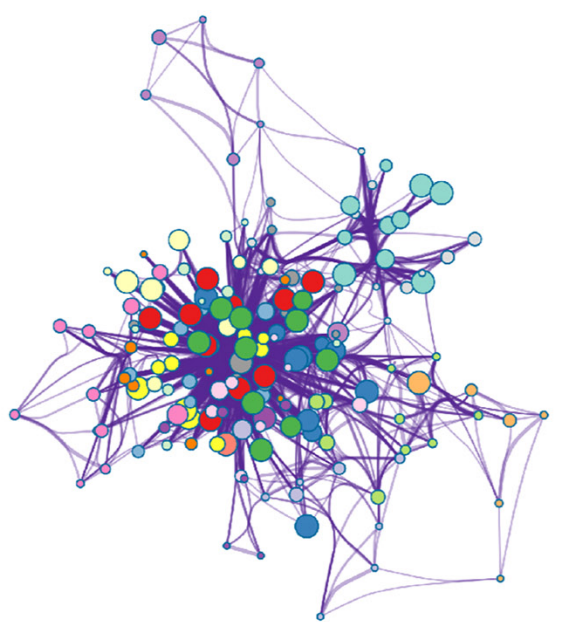

B

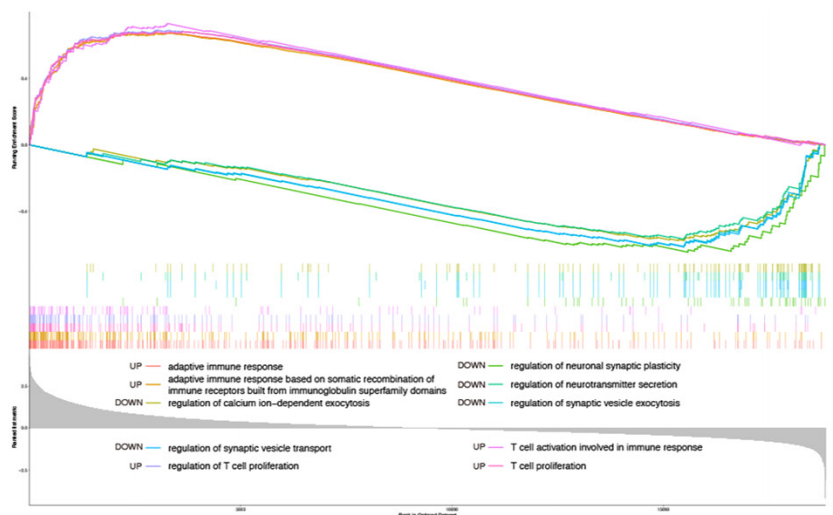

D

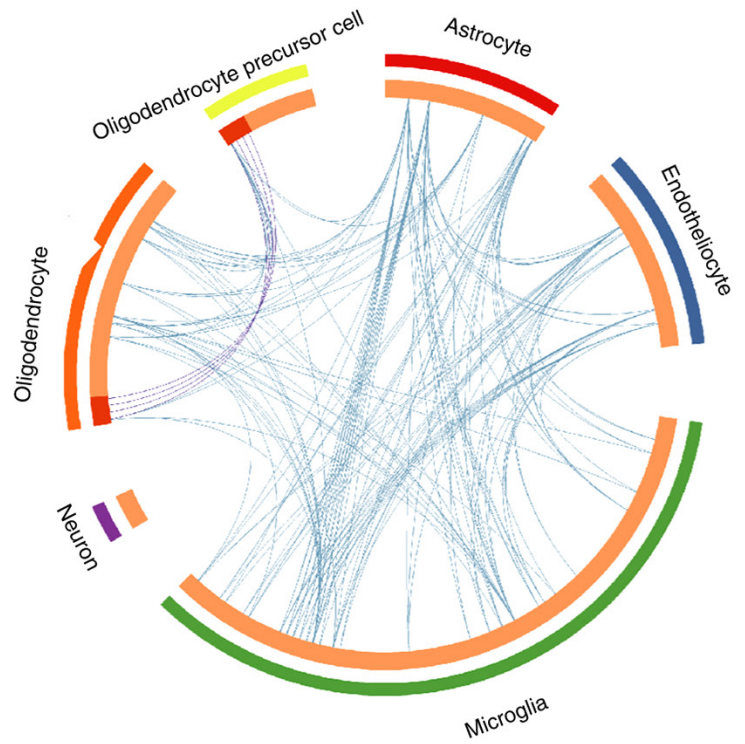

$\mathrm{F}$

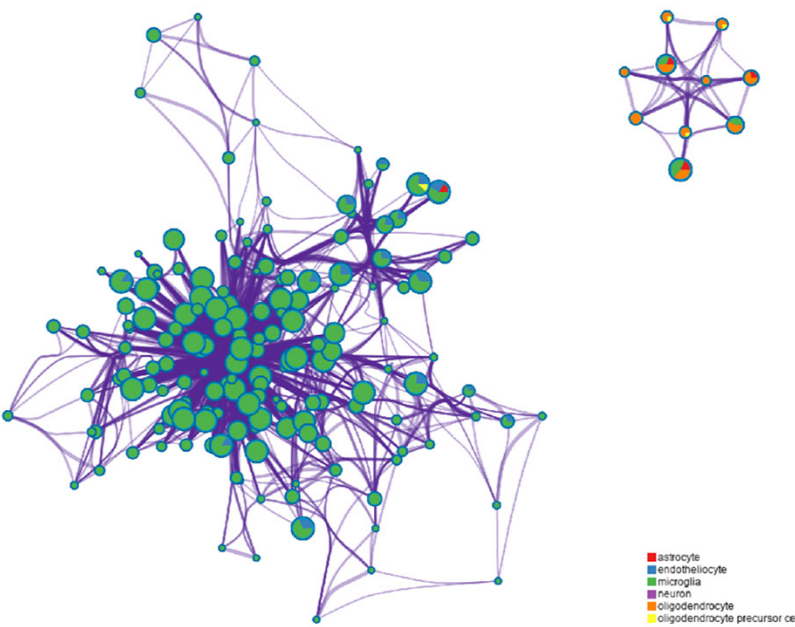

Figure 4. Bioinformatic analysis of AxD. (A) Volcano plot of gene expression. Red represents high expression, blue low expression and black no significant change in expression. (B) Enrichment plot of the top five significantly up- and downregulated biological processes by GSEA analysis. (C) Expression level of cell type-specific DEGs. The outside and inside arcs represent AxD and control groups, respectively. (D) Overlaps between specific cell types are shown in the Circos plot. The inner circle represents gene lists. Genes shared by different cell types are linked by purple lines, while different genes belonging to the same enriched terms are linked by blue lines. (E) Enrichment network of cell-specific DEGs. Each circular node represents an enriched term. The cluster identities and the number of enriched genes are indicated by the color and size of the node, respectively. $(\mathrm{F})$ Network of enriched terms. The color of each pie sector represents the involved cell types, and the area is proportional to the number of hits that are enriched in that specific term. GSEA, Gene Set Enrichment Analysis; DEGs, differentially expressed genes; AxD, Alexander disease.

were used for sample loading and transfer normalization. PVDF membranes with transferred proteins were incubated with primary antibodies at $4^{\circ} \mathrm{C}$ overnight. Then, blots were incubated with secondary HRP-conjugated antibodies (1:5,000; cat. no. A0208; Beyotime Institute of Biotechnology; cat. no. D110203; Sangon Biotech Co., Ltd.) for $60 \mathrm{~min}$ at 
room temperature and detected by SuperSignal ${ }^{\mathrm{TM}}$ Western Blot Enhancer (cat. no. 46641; Thermo Fisher Scientific, Inc.). Densitometry analysis of protein bands was performed using ImageJ software version 1.52p (National Institutes of Health).

For immunofluorescence, $48 \mathrm{~h}$ after transfection, 293T cells in 24-well plates were fixed with $4 \%$ paraformaldehyde for $30 \mathrm{~min}$ at room temperature, blocked with $10 \%$ normal donkey serum (cat. no. 017-000-121; Jackson ImmunoResearch Laboratories, Inc.) and 0.3\% Triton X-100 in PBS for $60 \mathrm{~min}$ at room temperature, and incubated with primary antibodies mentioned above to detect LC3 and LAMP-1 in blocking solution at $4^{\circ} \mathrm{C}$ overnight. Next, cells were stained with a Alexa Fluor ${ }^{\circledR}$ 594-conjugated secondary antibody $(1: 1,000$; cat. no. A-21442; Thermo Fisher Scientific, Inc.) for $60 \mathrm{~min}$ at room temperature, and nucleic acid was stained with DAPI (1:10,000; cat. no. 62248; Thermo Fisher Scientific, Inc.) for $5 \mathrm{~min}$ at room temperature. Cells were visualized under a Zeiss LSM 710 confocal microscope (magnification, $\mathrm{x} 40$; zoom, x2; Carl Zeiss AG).

Bioinformatics analysis. The GSE116327 expression profile dataset (21) sequenced on GPL16791 (Illumina HiSeq 2500; Illumina, Inc.) was downloaded from the Gene Expression Omnibus (GEO) database (https://www.ncbi.nlm.nih. gov/geo/). A total of five AxD and three normal post-mortem human brain samples were selected (Table SI). All tissues were frontal cortex tissues. Rosenthal fiber accumulation could be detected in the tissues of patients with $\mathrm{AxD}$, but not in those of normal controls (21).

Differential gene expression analysis was performed using the DESeq2 package (22). Adjusted (adj.)P $<0.05$ and the absolute value of $\log 2$ fold-change $>1.00$ were set as the threshold for differentially expressed genes (DEGs). The ggplot2 package was used to construct the volcano plot (23). The clusterProfiler package was used to perform Gene Set Enrichment Analysis (GSEA), basing the 'Biological Process' terms of the Gene Ontology (GOBP) database (24-26). The list of the top 100 cell type-specific genes for microglia, neurons, oligodendrocytes, oligodendrocyte precursor cells, astrocytes and endothelial cells was obtained from the study of McKenzie et al (27) (Table SII). Based on this list, cell type-specific DEGs were extracted from the selected data. The circlize package was used to visualize the expression levels of cell type-specific DEGs (28). Cell type-specific DEGs lists were uploaded to Metascape (metascape.org/; November 2020) (29) for functional and pathway enrichment analysis. Based on the Metascape online tool, different genes were linked in the circos plot if both were associated with the same function or pathway term. To demonstrate the association between these terms, a subset of significant representative terms from each of the 20 top-score clusters were selected ( $\leq 15$ terms/cluster; $\leq 250$ terms in total). Then the enriched terms were converted into a network layout by Metascape (29).

Statistical analysis. Results of the cellular experiments are presented as the mean \pm SD and were statistically analyzed using GraphPad Prism version 8.0.1 software (GraphPad Software, Inc.). One-way ANOVA was used to compare the expression levels of GFAP protein in different groups, followed by Tukey's post hoc test. A paired Student's t-test was used to analyze relative GFAP levels of each group after two different treatments (DMSO or BafAI. $\mathrm{P}<0.05$ was considered to indicate a statistically significant difference.

\section{Results}

Clinical findings. Patient P3433, a 29-year-old woman with no family history of $\mathrm{AxD}$, had been suffering from gait disturbance since the age of 10 . No issues with developmental retardation or psychomotor abnormalities had been recorded. She had been diagnosed with scoliosis at 14 years old (Fig. S1A), for which she had received corrective surgery (Fig. S1B). At the age of 20 years old, she was completely wheelchair-bound and began to suffer dysarthria, urinary dysfunction and aspiration pneumonia. Neurological examination revealed strabismus, movement disorders of extraocular muscle and bilaterally horizontal nystagmus. Muscle tension and strength of lower extremities were notably decreased. The bilateral pathological reflexes and finger-to-nose tests were positive. Cognitive function was normal. Laboratory examinations yielded unremarkable findings. A documented de novo mutation was identified in the GFAP gene (c.214G>A), which was absent in the $1000 \mathrm{~g}$, ExAC, gnomAD data and the controls (30). This mutation was also not detected in her healthy parents; the family pedigree is presented in Fig. 1A. c. $214 \mathrm{G}>\mathrm{A}$ was predicted to be damaging by PolyPhen 2 (probability score, 0.945), damaging by SIFT (score, 0.001) and disease-causing by MutationTaster (probability score, 1.000; data not shown). According to the ACMG guidelines, this variant was predicted as likely pathogenic.

Patient P4288 was a 33-year-old man without a family history of AxD. He began having gait disorders and external rotation of the left foot at the age of 31. Imaging examinations revealed thoracic spinal cord thinning and tethered cord syndrome. Surgical decompression of the cauda equina nerve was performed and symptoms were slightly improved following surgery. However, $\sim 1$ year later, the patient presented with poor coordination, spasticity and dysphagia, subsequently relying on a wheelchair. Physical examinations revealed speech disfluency and horizontal nystagmus. Muscle strength in the lower limbs was decreased, while muscle tension was significantly increased. Bilateral pathological reflexes were positive. Coordinated movement tests were unable to complete. Laboratory investigations were almost normal. The c. $1235 \mathrm{C}>\mathrm{T}$ variant was detected in the patient, which was absent in the $1000 \mathrm{~g}$, ExAC and gnomAD data, as well as the controls. In addition, this mutation was not identified in the patient's parents (Fig. 1B). SIFT (score, 0.001), PolyPhen-2 (score, 0.519) and MutationTaster (probability score, 1.000) predicted that the variants were damaging, possibly damaging and disease-causing, respectively (data not shown). According to the ACMG guidelines, the $\mathrm{c} .1235 \mathrm{C}>\mathrm{T}$ variant was classified as likely pathogenic.

To investigate the association between mutation sites and protein domains, previously reported GFAP mutations were summarised according to the Human Gene Mutation Database. A total of 135 mutations in the GFAP gene (NM_002055.5) had been previously reported, including 121 missense, one nonsense, one splicing, two regulatory, three small deletion, five small insertion and three small indel mutations (Fig. 2). 
Neuroimaging findings. The brain MRI scan of the two probands displayed white matter lesions in bilateral corona radiata, centrum semiovale and the regions surrounding the 4th ventricle (Fig. 1C and D). Volume estimation based on a white matter tissue probability map in CAT12 suggested $17.33 \mathrm{ml}$ white matter hyperintensity in patient P3433, while the TIV was $1,218.15 \mathrm{ml}$ (data not shown). Comparatively minor white matter lesions were observed in patient P4288.

The average TIV of subjects in the control group was $1,481.34 \mathrm{ml}$, with a standard deviation of $1,13.83 \mathrm{ml}$ (data not shown). After adjusting for TIV, patient P3433 exhibited atrophy following grey matter analysis, mainly in the bilateral putamen, thalamus and cerebellum. Atrophic white matter was observed in the corona radiata, centrum semiovale, cerebellopontine angle and medulla of the patient, which was consistent with leukodystrophy in AxD. Patient P4288 exhibited a similar pattern of atrophy in the white matter, while no significant grey matter differences were observed (Fig. 1E and F).

Regarding neural activity, a higher ALFF in the cerebellar vermis, cerebellopontine angles, occipital and posterior parietal cortex was observed in patient P3433. An increased DC distribution was observed in both the frontal and posterior parietal cortex, overlapping with ReHo mainly in the cerebellum and posterior cortex. In P4288, a higher ALFF, DC and ReHo overlapped in similar regions. An increased DC was also observed in the bilateral insula (Fig. $1 \mathrm{G}$ and $\mathrm{H}$ ).

Functional analysis. To explore the effects of GFAP variants on the protein level and localization, WT or MUT GFAP-GFP plasmids were transiently transfected into 293 cells. In the course of the experiment, it was noted that the levels of soluble GFAP in the c.1235G $>\mathrm{T}$ group were lower than those in the WT group. Correspondingly, the $\mathrm{c} .1235 \mathrm{G}>\mathrm{T}$ group exhibited a relatively higher level in the insoluble fraction. No significant difference in soluble or insoluble fractions was observed among the WT, c. $214 \mathrm{G}>\mathrm{A}$ and positive control groups (c.715C $>$ T) (Fig. 3A). Immunostaining results showed that the WT group exhibited diffuse distribution of GFAP proteins throughout the cytoplasm with a few aggregates, while MUT GFAP proteins appeared as punctate aggregations in perinuclear areas (Fig. 3B).

It was also detected whether aberrant GFAP accumulation was associated with the autophagy-lysosome pathway. As shown by immunofluorescence, MUT GFAP was clearly co-expressed with LC3 and lysosome (labeled by LAMP1; Fig. 3C and D). Next, the autophagic flux was detected using BafAI. Increased levels of LC3-II were observed in soluble MUT groups when they were treated with BafAI (Fig. 3E). However, the WT group also exhibited mild autophagy, since GFAP-WT overexpression could partly contribute to aggregate formation. Of note, under BafAI treatment, the soluble GFAP levels of MUT groups exhibited an increasing trend, particularly in the c.1235C $>\mathrm{T}$ and c.214G $>$ A groups (Fig. 3F).

Bioinformatics analysis. To investigate potential pathway changes in the pathology of $\mathrm{AxD}$, the RNA-sequencing data of patients with AxD and healthy controls were downloaded from the GEO database. Compared with the control brains, a total of 2,100 DEGs were detected in the AxD brains (Fig. 4A). The overall expression data were analyzed by GSEA based on the
GO-BP gene sets. A total of 1,315 up- and 125 downregulated 'Biological Process' pathways were identified in AxD samples compared with control samples (adj.P<0.05). The top five up- and downregulated gene sets are shown in Fig. 4B and revealed the upregulation of 'adaptive immune response', 'adaptative immune response based on somatic recombination of immune receptors built from immunoglobin superfamily domains', 'regulation of $\mathrm{T}$ cell proliferation', 'T cell activation involved in immune response' and ' $\mathrm{T}$ cell proliferation', and downregulation of 'regulation of calcium ion-dependent exocytosis', 'regulation of neuronal synaptic plasticity', 'regulation of neurotransmitter secretion', 'regulation of synaptic vesicle exocytosis' and 'regulation of synaptic vesicle transport' in AxD.

Furthermore, a set of brain cell consensus signatures were used to screen for cell-specific changes in AxD brain transcripts (27). Based on cell-specific gene lists, the expression levels of significant DEGs are shown in Fig. 4C. The overlap of functional terms among specific cell types is shown in a circos plot (Fig. 4D). A subset of the representative enriched terms from each of the top 20 clusters, including 'myeloid leukocyte activation', 'lymphocyte activation', 'phagocytosis' and 'signaling by interleukins', was converted into a network layout (Fig. 4E). Most terms of this network were associated with inflammatory-immune responses and formed closely functional networks. The details are shown in Table SIII. In addition, it was found that microglia may play a crucial role in the development of inflammatory processes, since the upregulated genes in microglia were mainly associated with 'leukocyte activation involved in immune response', 'leukocyte migration', 'lymphocyte activation', as well as the 'regulation of cytokine production' (Figs. 4F and S2A). Considering that astrocytes are mainly involved in AxD pathogenesis (31), astrocyte gene expression changes were compared between AxD states and healthy states. GO enrichment results of astrocytes showed that the GRM3 gene, the only downregulated cell-specific gene in astrocytes, was involved in 'synaptic signaling' (GO:0099536; data not shown). The upregulated genes in astrocytes were mainly associated with the 'regulation of protein catabolic process', 'spinal cord injury' and 'myeloid leukocyte activation' (Fig. S2B).

\section{Discussion}

In the present study, two cases of bulbospinal form $\mathrm{AxD}$ due to de novo GFAP mutations were described. It has been reported that $98 \%$ of patients with a clinical diagnosis of AxD carry a variant of GFAP, while the cause of $\mathrm{AxD}$ in the remaining $2 \%$ of patients remains unknown (32). GFAP $\alpha$ is the predominant isoform, which is the 432 amino acid protein that accounts for $90-95 \%$ of the total GFAP protein in the human central nervous system $(1,33)$. The other GFAP isoforms, such as GFAP $\beta, \delta$ and $\kappa$, derive from alternative RNA start sites (4). To date, GFAP $\alpha$ is the subject of most published studies $(4,33)$. The GFAP $\alpha$ protein comprises a central $\alpha$-helical rod domain flanked with the non-helical N-terminal head and C-terminal tail domains, which are important for assembling into the cellular IF (diameter, $10 \mathrm{~nm}$ ) (4). The rod domain is divided into four $\alpha$-helical segments (1A and B, and 2A and B) and exhibits higher conservation (8). Pathogenic mutations are 
scattered all over the GFAP protein domains, but are more abundant in the $1 \mathrm{~A}$ and $2 \mathrm{~B}$ segments of the rod domain.

However, the clinical severity of AxD varies markedly and the genotype-phenotype correlation is complicated. The variants affecting the hot-spot amino acids R79, R88, R239 and R416 account for $>50 \%$ of mutations identified in patients with AxD, while R79, R88 and R239 mutations are common in infantile and juvenile forms $\mathrm{AxD}(8,30)$. In contrast to these typical relations, the phenotype correlations of numerous other mutations are poorly understood. There exists a variety of clinical presentations in AxD, even among individuals carrying the same mutation. For example, the R416W variation can be found in all three forms of $\mathrm{AxD}$ (8). In addition, it has been found that individuals carrying the same mutation, such as D78E, S247P, L331P and D417A, show clinical variability, with mixed infantile-adult or juvenile-adult manifestations (1,34,35). Patient P3433 with the c. $214 \mathrm{G}>\mathrm{A}$ mutation in the present study exhibited similar symptoms to those of adult-onset form, but at a juvenile-onset age, which may be linked to the fact that the variant is located near R79 and D78. Patient P4288 carrying the c.1235C $>\mathrm{T}$ mutation in the C-terminal domain exhibited typical adult-onset symptoms, which rapidly progressed to severe manifestations. Compared with R416 mutations, these findings were consistent with previous reports that mutations in the tail domain can have varied clinical courses and severities $(8,36)$. The cause of these variations remains unclear. Genetic modifiers or environmental impactors may affect clinical phenotypes (1).

A novel analysis method based on the fMRI data was used in the present study to explore the atrophic pattern and spontaneous brain functional network of AxD. A similar pattern of white matter atrophy was found in two patients, with involvements of the medulla and periventricular regions. The subventricular region has been reported to be the most vulnerable to the pathogenesis of $\mathrm{AxD}$ (37). In total, $1 / 3$ of patients with AxD display abnormal signals in the periventricular rim, which may be associated with the abnormal aggregation of Rosenthal fibers in subependymal regions $(38,39)$. Of note, in the current study patient P3433 with major white matter damages also presented grey matter atrophy, mainly in the bilateral putamen and thalamus, which was first reported in AxD. Grey matter volume loss may be linked to long-term disability $(40,41)$, which could explain the grey matter atrophy in patient P3433. Furthermore, several mechanisms may underlie grey matter damage, including iron deposition, mitochondrial failure, white matter lesion-induced retrograde degeneration and meningeal inflammation $(42,43)$. To the best of our knowledge, the present study was the first to evaluate the neural activities and regional connections in these patients through three different types of data-driven analysis: ReHo, DC and ALFF. The results showed increased ReHo, DC and ALFF overlapping in the cerebellum and posterior parietal cortex, indicating a higher amount of neural communication among these regions than controls. The cerebellum, as part of certain largescale networks, participates in communicating with association areas, such as the frontal lobe and posterior parietal cortex (44). Severe atrophies of the white or grey matter could result in the overload and collapse of brain networks (40). Increased connectivity in these regions may be a type of compensatory mechanism or reorganization of the brain network for this disease. Considering the very small sample size of AxD in the present study, more clinical studies are required to reach correlational conclusions and explore the underlying mechanisms.

It is commonly known that the best-known pathology in AxD is the accumulation of mutant GFAP (31). It is noteworthy that the solubility of GFAP variant c.1235C $>\mathrm{T}$ was significantly decreased in the present study, similar to the performance of variant c. $1178 \mathrm{G}>\mathrm{T}$ and c.1246C $>\mathrm{T}(45,46)$. These mutations are located in the tail domain, which is highly conserved and important for stabilizing filament-filament interactions (47). Filament disorganization may enhance the stability of the assembled protein, which could result in increased resistance to salt extraction, and a declined solubility of GFAP (48). Further studies need to examine the detailed mechanism of the tail domain that facilitates the assembly of GFAP. Furthermore, the overlap of LC3 with abnormal GFAP accumulation was clearly observed in the present study. Aggregate-prone proteins unsuccessfully corrected by chaperones are generally ubiquitylated and subsequently recognized by protein degrading pathways, such as the ubiquitin-proteasome system and the autophagy-lysosomal pathway (49). It has been demonstrated that mutant GFAP produces a strong inhibition of proteasome activity and leads to decreased protein turnover rates $(50,51)$. In the present study, the degradation of GFAP aggregates was accompanied by LC3-II upregulation, suggesting that the autophagy pathway may act as a compensatory mechanism for degrading aggregates in $\mathrm{AxD}$ (52). Nevertheless, it remains to be explored whether any other potential pathways are associated with GFAP degradation. Collectively, these findings verified the disease-causing of the variants studied herein and supported that GFAP mutations can be distinguished by mutant aggregates and the upregulation of autophagy.

To further explore the potential pathogenic mechanisms of $\mathrm{AxD}$, transcriptional alterations in $\mathrm{AxD}$ brains were investigated. Bioinformatics analysis of gene expression profiles revealed the involvement of inflammatory immune-related reactions in $\mathrm{AxD}$. It has been demonstrated that $\mathrm{AxD}$ astrocytes sustain a state of cellular stress caused by abnormal aggregates and act as origins of pathology (31). Microglia changes may directly result from chemokines released by activated astrocytes, while damage-associated molecular patterns, such as small heat shock proteins, which markedly accumulate in AxD astrocytes, could also function in microglia alterations $(2,53)$. It is possible that the inflammatory responses are due not only to astrocyte stress, but also to the reactions of dysfunctional astrocytes to external stimulations from other cells, particularly activated microglia (31). Consistent with the present findings, dysfunctional astrocytes are less able to maintain the ion transport, synaptic transmission and neurotransmitter homeostasis required for normal cell-cell communications (31), thereby playing an important role in inflammation alongside microglia. In AxD, astrocyte-derived molecules also inhibit oligodendrocyte progenitor cell function and myelination formation (21). These disruptions of brain homeostasis, in turn, influence astrocyte phenotypes and contribute to inflammation, creating a vicious circle. However, the nature of these interactions and their 
consequences are unclear, and future studies are required to provide novel insights into mechanistic investigations for AxD.

Although AxD has not been acknowledged as an inflammatory disease, several studies have revealed a marked inflammatory environment in both mice and patients with AxD $(21,54,55)$. Transgene of WT human GFAP $\left(G F A P^{\mathrm{Tg}}\right)$ mice and heterozygous $\mathrm{R} 236 \mathrm{H}$ knock-in mutation lines crossing with the $G F A P^{\mathrm{Tg}}$ lines $\left(G F A P^{\mathrm{Tg}} / \mathrm{R} 236 \mathrm{H}^{+/-}\right)$mice exhibit Rosenthal fibers, particularly in the hippocampus, corpus callosum, olfactory bulbs, subpial tissues and periventricular regions, more closely resembling adult-onset $\mathrm{AxD}$ than infantile AxD $(56,57)$. Studies have reported clearly upregulated inflammatory processes in the hippocampus and spinal cord of $G F A P^{\mathrm{Tg}} / \mathrm{R} 236 \mathrm{H}^{+/-}$mice, and olfactory bulb of $G F A P^{\mathrm{Tg}}$ mice $(54,58)$. Furthermore, activated inflammatory responses in the brainstem and spinal cord have also been reported in infantile- and juvenile-form patients (54). Accordingly, inflammation may be associated with all three forms of the disease. We hypothesized that inflammatory responses may also occur in these central nervous system tissues in patients with bulbospinal form, particularly in the brainstem and spinal cord, as these regions are particularly affected in these patients. However, no data from the brainstem or cerebellar regions of patients with bulbospinal form were available to be analyzed, and the transcriptional data in the present study were derived from the frontal lobe cortex of patients with infantile form. Future research should focus on the specific brain regions of different subtypes to obtain more detailed findings.

In conclusion, two de novo variants of GFAP (c.214G>A and $c .1235 \mathrm{C}>\mathrm{T}$ ) were identified in patients with $\mathrm{AxD}$ from unrelated families. The functional analysis provided essential evidence revealing the pathogenicity of the identified variants. Increased brain functional connectivity in the cerebellum and posterior parietal cortex was observed in two probands, and grey matter atrophy in the patient with the more severe white matter damage. It was concluded that these changes might be a type of compensatory mechanism or reorganization of the collapsed brain network in AxD. Bioinformatics analysis further indicated that inflammatory immune-related responses play a critical role in $\mathrm{AxD}$. These findings not only broadened the clinical and genetic spectrums of $\mathrm{AxD}$, but also provided an important basis for the study of its pathogenic mechanism.

\section{Acknowledgements}

Not applicable.

\section{Funding}

The present study was supported by the National Natural Science Foundation of China (grant nos. 81870889, 81571086 and 82071258), Ministry of Science and Technology of the People's Republic of China (grant nos. 2017YFC1310200 and 2016YFC1305804), Shanghai Municipal Education Commission-Gaofeng Clinical Medicine Grant Support (grant no. 20161401), Shanghai Municipal Planning Commission of Science and Research Fund for Youth (grant no. 20184Y0358) and Interdisciplinary Project of Shanghai Jiao Tong University (grant no. YG2016MS64).

\section{Availability of data and materials}

The datasets used and/or analyzed during the current study are available from the corresponding author on reasonable request.

\section{Authors' contributions}

XS, JJ and LC conceived and designed the study. XS performed the experiments and processed the data. BL and HT were responsible for MRI data acquisition, preprocessing and statistical analysis. WT and FZ performed genetic analysis. ZZ performed bioinformatic analysis. XS, JJ, HT and LC confirmed the authenticity of all the raw data. XS and JJ wrote the manuscript. JJ produced figures and tables. BL, HT and LC reviewed and edited the manuscript. All authors read and approved the final manuscript.

\section{Ethics approval and consent to participate}

This study was approved by the Ethics Committee of Rui Jin Hospital, Shanghai Jiao Tong University School of Medicine (approval no. 2019-153; Shanghai, China). All participants provided informed consent for participation.

\section{Patient consent for publication}

Written informed consent was obtained from all participants involved in this study.

\section{Competing interests}

The authors declare that they have no competing interests.

\section{References}

1. Messing A: Alexander disease. Handb Clin Neurol 148: 693-700, 2018.

2. Iwaki T, Kume-Iwaki A, Liem RK and Goldman JE: $\alpha$ B-crystallin is expressed in non-lenticular tissues and accumulates in Alexander's disease brain. Cell 57: 71-78, 1989.

3. Brenner M, Johnson AB, Boespflug-Tanguy O, Rodriguez D, Goldman JE and Messing A: Mutations in GFAP, encoding glial fibrillary acidic protein, are associated with Alexander disease. Nat Genet 27: 117-120, 2001.

4. Hol EM and Capetanaki Y: Type III Intermediate Filaments Desmin, Glial Fibrillary Acidic Protein (GFAP), Vimentin, and Peripherin. Cold Spring Harb Perspect Biol 9: a021642, 2017.

5. Li R, Messing A, Goldman JE and Brenner M: GFAP mutations in Alexander disease. Int J Dev Neurosci 20: 259-268, 2002.

6. Quinlan RA, Brenner M, Goldman JE and Messing A: GFAP and its role in Alexander disease. Exp Cell Res 313: 2077-2087, 2007.

7. Jones JR, Kong L, Hanna MG IV, Hoffman B, Krencik R, Bradley R, Hagemann T, Choi J, Doers M, Dubovis M, et al: Mutations in GFAP Disrupt the Distribution and Function of Organelles in Human Astrocytes. Cell Rep 25: 947-958.e4, 2018.

8. Li R, Johnson AB, Salomons G, Goldman JE, Naidu S, Quinlan R, Cree B, Ruyle SZ, Banwell B, D'Hooghe M, et al: Glial fibrillary acidic protein mutations in infantile, juvenile, and adult forms of Alexander disease. Ann Neurol 57: 310-326, 2005.

9. Yoshida T, Sasaki M, Yoshida M, Namekawa M, Okamoto Y, Tsujino S, Sasayama H, Mizuta I and Nakagawa M; Alexander Disease Study Group in Japan: Nationwide survey of Alexander disease in Japan and proposed new guidelines for diagnosis. J Neurol 258: 1998-2008, 2011. 
10. Johnson AB and Brenner M: Alexander's disease: Clinical, pathologic, and genetic features. J Child Neurol 18: 625-632, 2003.

11. Miller SA, Dykes DD and Polesky HF; MWer S: A simple salting out procedure for extracting DNA from human nucleated cells. Nucleic Acids Res 16: 1215, 1988 .

12. Li H and Durbin R: Fast and accurate short read alignment with Burrows-Wheeler transform. Bioinformatics 25: 1754-1760, 2009.

13. McKenna A, Hanna M, Banks E, Sivachenko A, Cibulskis K, Kernytsky A, Garimella K, Altshuler D, Gabriel S, Daly M, et al: The Genome Analysis Toolkit: A MapReduce framework for analyzing next-generation DNA sequencing data. Genome Res 20: 1297-1303, 2010.

14. Wang K, Li M and Hakonarson H: ANNOVAR: Functiona annotation of genetic variants from high-throughput sequencing data. Nucleic Acids Res 38: e164-e164, 2010.

15. Richards S, Aziz N, Bale S, Bick D, Das S, Gastier-Foster J, Grody WW, Hegde M, Lyon E, Spector E, et al; ACMG Laboratory Quality Assurance Committee: Standards and guidelines for the interpretation of sequence variants: A joint consensus recommendation of the American College of Medical Genetics and Genomics and the Association for Molecular Pathology. Genet Med 17: 405-424, 2015.

16. Yan CG, Wang XD, Zuo XN and Zang YF: DPABI: Data Processing \& Analysis for (Resting-State) Brain Imaging. Neuroinformatics 14: 339-351, 2016.

17. Yang H, Long XY, Yang Y, Yan H,Zhu CZ, Zhou XP,Zang YF and Gong QY: Amplitude of low frequency fluctuation within visual areas revealed by resting-state functional MRI. Neuroimage 36 : 144-152, 2007

18. Eijlers AJ, Meijer KA, Wassenaar TM, Steenwijk MD, Uitdehaag BM, Barkhof F, Wink AM, Geurts JJ and Schoonheim MM: Increased default-mode network centrality in cognitively impaired multiple sclerosis patients. Neurology 88 : 952-960, 2017

19. Zang Y, Jiang T, Lu Y, He Y and Tian L: Regional homogeneity approach to fMRI data analysis. Neuroimage 22: 394-400, 2004

20. Bresciani A, Spiezia MC, Boggio R, Cariulo C, Nordheim A, Altobelli R, Kuhlbrodt K, Dominguez C, Munoz-Sanjuan I, Wityak J, et al: Quantifying autophagy using novel LC3B and p62 TR-FRET assays. PLoS One 13: e0194423, 2018.

21. Li L, Tian E, Chen X, Chao J, Klein J, Qu Q, Sun G, Sun G, Huang Y, Warden CD, et al: GFAP Mutations in Astrocytes Impair Oligodendrocyte Progenitor Proliferation and Myelination in an hiPSC Model of Alexander Disease. Cell Stem Cell 23: 239-251. e6, 2018.

22. Love MI, Huber W and Anders S: Moderated estimation of fold change and dispersion for RNA-seq data with DESeq2. Genome Biol 15: 550, 2014

23. Wickham H: ggplot2. Wiley Interdiscip Rev Comput Stat 3 : 180-185, 2011

24. Subramanian A, Tamayo P, Mootha VK, Mukherjee S, Ebert BL, Gillette MA, Paulovich A, Pomeroy SL, Golub TR, LanderES, et al: Gene setenrichment analysis: A knowledge-based approach for interpreting genome-wide expression profiles. Proc Natl Acad Sci USA 102: 15545-15550, 2005.

25. Yu G, Wang L-G, Han Y and He Q-Y: clusterProfiler: An R package for comparing biological themes among gene clusters. OMICS 16: 284-287, 2012

26. Ashburner M, Ball CA, Blake JA, Botstein D, Butler H, Cherry JM, Davis AP, Dolinski K, Dwight SS, Eppig JT, et al; The Gene Ontology Consortium: Gene ontology: Tool for the unification of biology. Nat Genet 25: 25-29, 2000.

27. McKenzie AT, Wang M, Hauberg ME, Fullard JF, Kozlenkov A Keenan A, Hurd YL, Dracheva S, Casaccia P, Roussos P, et al: Brain cell type specific gene expression and co-expression network architectures. Sci Rep 8: 1-19, 2018.

28. Gu Z, Gu L, Eils R, Schlesner M and Brors B: circlize Implements and enhances circular visualization in R. Bioinformatics 30 : $2811-2812,2014$

29. Zhou Y, Zhou B, Pache L, Chang M, Khodabakhshi AH, Tanaseichuk O, Benner C and Chanda SK: Metascape provides a biologist-oriented resource for the analysis of systems-level datasets. Nat Commun 10: 1-10, 2019.

30. Prust M, Wang J, Morizono H, Messing A, Brenner M, Gordon E, Hartka T, Sokohl A, Schiffmann R, Gordish-Dressman H, et al: GFAP mutations, age at onset, and clinical subtypes in Alexander disease. Neurology 77: 1287-1294, 2011.

31. Olabarria M and Goldman JE: Disorders of Astrocytes: Alexander Disease as a Model. Annu Rev Pathol 12: 131-152, 2017.
32. Yoshida T: Clinical characteristics of Alexander disease. Neurodegener Dis Manag 10: 325-333, 2020.

33. Messing A and Brenner M: GFAP at 50. ASN Neuro 12 1759091420949680,2020

34. Stumpf E, Masson H, Duquette A, Berthelet F, McNabb J, Lortie A, Lesage J, Montplaisir J, Brais B and Cossette P: Adult Alexander disease with autosomal dominant transmission: A distinct entity caused by mutation in the glial fibrillary acid protein gene. Arch Neurol 60: 1307-1312, 2003.

35. Messing A, Brenner M, Feany MB, Nedergaard M and Goldman JE: Alexander disease. J Neurosci 32: 5017-5023, 2012

36. Kinoshita T, Imaizumi T, Miura Y, Fujimoto H, Ayabe M, Shoji H, Okamoto Y, Takashima H, Osame M and Nakagawa M A case of adult-onset Alexander disease with Arg416Trp human glial fibrillary acidic protein gene mutation. Neurosci Lett 350: 169-172, 2003

37. Sawaishi Y: Review of Alexander disease: Beyond the classical concept of leukodystrophy. Brain Dev 31: 493-498, 2009.

38. Balbi P, Salvini S, Fundarò C, Frazzitta G, Maestri R, Mosah D, Uggetti $\mathrm{C}$ and Sechi G: The clinical spectrum of late-onset Alexander disease: A systematic literature review. J Neurol 257: 1955-1962, 2010

39. van der Knaap MS, Ramesh V, Schiffmann R, Blaser S, Kyllerman M, Gholkar A, Ellison DW, van der Voorn JP, van Dooren SJ, Jakobs C, et al: Alexander disease: Ventricular garlands and abnormalities of the medulla and spinal cord. Neurology 66: 494-498, 2006.

40. Eshaghi A, Prados F, Brownlee WJ, Altmann DR, Tur C, Cardoso MJ, De Angelis F, van de Pavert SH, Cawley N, De Stefano N, et al; MAGNIMS study group: Deep gray matter volume loss drives disability worsening in multiple sclerosis. Ann Neurol 83: 210-222, 2018.

41. Roosendaal SD, Bendfeldt $\mathrm{K}$, Vrenken $\mathrm{H}$, Polman $\mathrm{CH}$, Borgwardt S, Radue EW, Kappos L, Pelletier D, Hauser SL, Matthews PM, et al: Grey matter volume in a large cohort of MS patients: Relation to MRI parameters and disability. Mult Scler 17: 1098-1106, 2011.

42. Eshaghi A, Marinescu RV, Young AL, Firth NC, Prados F, Jorge Cardoso M, Tur C, De Angelis F, Cawley N, Brownlee WJ, et al: Progression of regional grey matter atrophy in multiple sclerosis. Brain 141: 1665-1677, 2018.

43. Calabrese M, Magliozzi R, Ciccarelli O, Geurts JJ, Reynolds R and Martin R: Exploring the origins of grey matter damage in multiple sclerosis. Nat Rev Neurosci 16: 147-158, 2015.

44. Ramnani N: Frontal lobe and posterior parietal contributions to the cortico-cerebellar system. Cerebellum 11: 366-383, 2012.

45. Chen YS, Lim SC, Chen MH, Quinlan RA and Perng MD: Alexander disease causing mutations in the $\mathrm{C}$-terminal domain of GFAP are deleterious both to assembly and network formation with the potential to both activate caspase 3 and decrease cell viability. Exp Cell Res 317: 2252-2266, 2011.

46. Der Perng M, Su M, Wen SF, Li R, Gibbon T, Prescott AR, Brenner M, and Quinlan RA: The Alexander disease-causing glial fibrillary acidic protein mutant, R416W, accumulates into Rosenthal fibers by a pathway that involves filament aggregation and the association of alpha B-crystallin and HSP27. Am J Hum Genet 79: 197-213, 2006.

47. Yoshida T, Sasayama $\mathrm{H}$ and Nakagawa $\mathrm{M}$ : The process of inducing GFAP aggregates in astrocytoma-derived cells is different between R239C and R416W mutant GFAP. A time-lapse recording study. Neurosci Lett 458: 11-14, 2009.

48. Hsiao VC, Tian R, Long H, Der Perng M, Brenner M, Quinlan RA and Goldman JE: Alexander-disease mutation of GFAP causes filament disorganization and decreased solubility of GFAP J Cell Sci 118: 2057-2065, 2005.

49. Kwon YT and Ciechanover A: The Ubiquitin Code in the Ubiquitin-Proteasome System and Autophagy. Trends Biochem Sci 42: 873-886, 2017.

50. Tang G, Xu Z and Goldman JE: Synergistic effects of the SAPK/JNK and the proteasome pathway on glial fibrillary acidic protein (GFAP) accumulation in Alexander disease. J Biol Chem 281: 38634-38643, 2006.

51. Tang G, Perng MD, Wilk S, Quinlan R and Goldman JE: Oligomers of mutant glial fibrillary acidic protein (GFAP) Inhibit the proteasome system in alexander disease astrocytes, and the small heat shock protein alphaB-crystallin reverses the inhibition. J Biol Chem 285: 10527-10537, 2010.

52. Tang G, Yue Z, Talloczy Z, Hagemann T, Cho W, Messing A, Sulzer DL and Goldman JE: Autophagy induced by Alexander disease-mutant GFAP accumulation is regulated by $\mathrm{p} 38 / \mathrm{MAPK}$ and mTOR signaling pathways. Hum Mol Genet 17: 1540-1555, 2008 
53. Singhal G, Jaehne EJ, Corrigan F, Toben C and Baune BT: Inflammasomes in neuroinflammation and changes in brain function: A focused review. Front Neurosci 8: 315, 2014.

54. Olabarria M, Putilina M, Riemer EC and Goldman JE: Astrocyte pathology in Alexander disease causes a marked inflammatory environment. Acta Neuropathol 130: 469-486, 2015.

55. Kora K, Kato T, Ide M, Tanaka T and Yoshida T: Inflammatory neuropathology of infantile Alexander disease: A case report. Brain Dev 42: 64-68, 2020

56. Hagemann TL, Connor JX and Messing A: Alexander disease-associated glial fibrillary acidic protein mutations in mice induce Rosenthal fiber formation and a white matter stress response. J Neurosci 26: 11162-11173, 2006.
57. Minkel HR, Anwer TZ, Arps KM, Brenner M and Olsen ML: Elevated GFAP induces astrocyte dysfunction in caudal brain regions: A potential mechanism for hindbrain involved symptoms in type II Alexander disease. Glia 63: 2285-2297, 2015.

58. Hagemann TL, Gaeta SA, Smith MA, Johnson DA, Johnson JA and Messing A: Gene expression analysis in mice with elevated glial fibrillary acidic protein and Rosenthal fibers reveals a stress response followed by glial activation and neuronal dysfunction. Hum Mol Genet 14: 2443-2458, 2005.

cc) (i) () $९$ This work is licensed under a Creative Commons

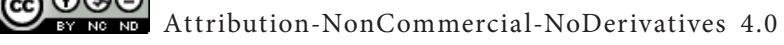
International (CC BY-NC-ND 4.0) License. 